\title{
Evaluación socioeconómica y ambiental de tres tipos de sistemas agroforestales en el Trópico Seco Nicaragüense
}

\begin{abstract}
Byron Moran Moreno ${ }^{1}$
Alejandrina Herrera ${ }^{2}$

Kenny López Benavides ${ }^{3}$
\end{abstract}

\section{RESUMEN}

En Nicaragua diferentes organizaciones con financiamiento externo y durante muchos años han promovido sistemas agroforestales. Este estudio evalúa tres tipos de sistemas agroforestales 6 años después de haber sido promovidos por el programa socio ambiental y desarrollo forestal (POSAF), fase 2006-2007; se clasifican e identifican los aportes socioeconómicos, ambientales y tecnologías con mayor adopción. Se estudiaron 61 unidades productivas, ubicadas en la reserva natural Tisey-Estanzuela, parte alta de la subcuenca del río Estelí, trópico seco de Nicaragua. Los resultados muestran que estadísticamente hay una agrupación de tres sistemas agroforestales, que coindice con la clasificación realizada desde la planificación del programa, pero indistintamente del sistema, se promovió y ejecutó tecnologías agroforestales similares. El ingreso agropecuario por unidad productiva mostró diferencias significativas $(\mathrm{p}=0.0119)$ en sistemas agrosilvícolas $(\mathrm{SAF})$, siendo el valor medio mensual menor (U\$103.59 \pm 30.83 dólares/mes) que en sistemas silvopastoril y de manejo de bosque. La mayor cantidad de cabezas de ganado fue estadísticamente diferente $(p=0.012)$ en sistemas manejo de bosque con regeneración natural más pasto (MBRNP) que en los otros dos sistemas agroforestales. Las tecnologías con mayor éxito y adopción fueron: cercas vivas, establecimiento de frutales, regeneración natural; manejo y conservación de bosques. Los principales aportes ambientales identificados fueron: sombra, disminución de la erosión del suelo, conservación de humedad y división de fincas. En conclusión, los productores agrupados en sistema agroforestal manejo de bosque con regeneración natural más pasto, presentaron los mayores aportes socioeconómicos en ingreso agropecuario y mayor cantidad de cabezas de ganado, pero hay mayor eficiencia en la producción de leche/ vaca/día en sistemas silvopastoriles. Económicamente las familias con sistemas agrosilvícolas se clasifican de subsistencia y dependen más de ingresos económicos externos a la finca. Los productores agrupados en sistema silvopastoril presentaron los mayores niveles de éxito y adopción de tecnologías agroforestales en: cercas vivas, áreas con frutales, cantidad de diques, áreas de pasto y banco forrajero.

Palabras Claves: Sistemas agroforestales, aportes socioeconómicos, unidades productivas, trópico seco, POSAF.

1 UNAN-Managua/FAREM-Estelí. Estación Experimental para el Estudio del Trópico Seco Correo Electrónico: byron.moran4@gmail.com 2 UNAN-Managua/FAREM-Estelí. Estación Experimental para el Estudio del Trópico Seco. Correro Electrónico: alejandjes@gmail.com 3 UNAN-Managua/FAREM-Estelí. Estación Experimental para el Estudio del Trópico Seco. Correro Electrónico: kenny.lb@hotmail.com 


\title{
Socio-economic and environmental evaluation of three types of agroforestry systems in the Nicaraguan Dry Tropics
}

\author{
Byron Moran Moreno ${ }^{1}$ \\ Alejandrina Herrera ${ }^{2}$ \\ Kenny López Benavides ${ }^{3}$
}

\begin{abstract}
In Nicaragua different organizations with external funding, and for many years, have promoted agroforestry systems. This study evaluates three types of agroforestry systems six years after being promoted by the socio-environmental and forestry development program (POSAF) phase 20062007; socio-economic, environmental and technology contributions adopted the most are classified and identified. Sixty one production units located in the natural reserve Tisey-Estanzuela, upper part of the river basin of Estelí, dry tropics of Nicaragua were studied. The results show that statistically there is a grouping of three agroforestry systems, which coincides with the classification made from the planning of the program, but regardless of the system, similar agroforestry technologies were promoted and executed. The agricultural income per farm showed significant differences $(\mathrm{p}=0.0119)$ in agroforestry systems (AFS), being the average monthly value lower (U \$ 103.59 \pm 30.83 dollars / month) than in silvopasture and forest management systems. Most cattle was statistically different $(\mathrm{p}=0.012)$ in systems of natural management with regeneration of forest with more grass (MBRNP) than in the other two agroforestry systems. The most successful and adopted technologies were: hedges, planting of fruit trees, natural regeneration; management and conservation of forests. The main environmental contributions identified were: shadow, reduced soil erosion, moisture conservation and division of property. In conclusion, producers grouped in forest management in agroforestry systems with natural regeneration plus grass, had the highest socioeconomic contributions in agricultural income and with foremost cattle, but there is more efficiency in milk production / cow / day in silvopastoral systems. Economically, families with agroforestry systems are classified as subsistence and depend more on external incomes apart from the farm. The producers grouped in silvopastoral system had the highest levels of success and in the adoption of agroforestry technologies: hedges, areas with fruits, number of ditches, grazing areas and fodder banks.
\end{abstract}

Keywords: Agroforestry, socioeconomic contributions, productive units, tropical dry POSAF.

1 UNAN-Managua/FAREM-Estelí.Estación Experimental station for the study of the dry tropics. E-mail: byron.moran4@gmail.com 2 UNAN-Managua/FAREM-Estelí. Experimental station for the study of the dry tropics. E-mail: alejandjes@gmail.com 3 UNAN-Managua/FAREM-Estelí. Experimental station for the study of the dry tropics. E-mail: kenny.lb@hotmail.com 


\section{INTRODUCCIÓN}

América Central y el Caribe están afrontando uno de los mayores retos en cuanto al uso de tierras rurales, la deforestación, la escasez de productos forestales y la degradación ambiental en tierras agrícolas frágiles bajo presión poblacional. Una de las repuestas al problema ha sido la agroforestería (Current D. et ál. 1995:3). Según (FAO, 2008) los sistemas agroforestales son un conjunto de tecnologías de manejo de suelo, agua, nutrientes y vegetación, que incluyen la gestión del suelo, manejo agronómico del cultivo y manejo forestal en los sistemas productivos. Los sistemas agroforestales se caracterizan por su estructura (naturaleza y arreglo del componente), función, usos y beneficios (Nair 1989; 1997; Somarriba 1998; Andrade 2002).

La agroforestería, como un sistema sostenible de uso de la tierra, precisamente se adapta en la estrategia de desarrollo sostenible. Las tecnologías agroforestales son herramientas promisorias dentro de este enfoque para mejorar el bienestar de la población rural y la conservación de su base de recursos. La agroforestería puede contribuir a reducir la tasa de deforestación, mantener la biodiversidad, mantener la integridad de las cuencas y la estabilidad del clima (Krishnamurthy, Ávila, 1999). (Toruño P. J. et al. 2012:5), menciona que los principales beneficios después del establecimiento de sistemas adaptativos como SAF son: incremento de la producción, restauración de los ecosistemas, recuperación de la biodiversidad, reducción de la erosión y reducción de la contaminación de las aguas.

Desde su estudio, las actividades regionales de agroforestería han tenido una documentación muy pobre, los esfuerzos para monitorear los proyectos han sido débiles o inexistentes (Current, et ál, 1995). Por su parte (Mendieta \& Rocha, 2007) revelan que en América Central no se han llevado a cabo investigaciones sistemáticas en agroforestería. Sin embargo, varias técnicas de mezclas de árboles con cultivos alimenticios fueron bien conocidas por los indios precolombinos, particularmente la práctica de agricultura migratoria, los huertos caseros, la mezcla de árboles y cultivos a lo largo de zanjas. Pero muchas de estas combinaciones todavía no están bien documentadas.

Diferentes organizaciones en Nicaragua con financiamiento externo y durante muchos años han promoviendo este tipo de sistemas, con el fin de hacer más integral la producción, tener mayor cantidad de alimentos, ingresos y generar otros bienes y servicios ambientales (FAO/PESA, 2010). La diversificación a través de los sistemas agroforestales podría brindar a los agricultores ventajas como: mejor uso de los recursos disponibles, mayor ingreso económico, menos dependencia de un solo cultivo y aumento de la disponibilidad de alimentos. (Lundgren y Raintree, 1983) afirman que muchas tecnologías son capaces de aumentar la productividad, pero cuestiona, ¿son sostenibles?, de igual manera hay numerosas tecnologías para la conservación de los recursos, pero ¿son productivas?. La agroforestería ha demostrado un potencial significativo para lograr ambos objetivos simultáneamente.

El programa socioambiental y desarrollo forestal (MARENA-POSAF), promovió e implementó en diversas zonas de Nicaragua, el manejo integrado de cuencas y reconversión de áreas agropecuarias convencionales por sistemas amigables con el medio ambiente, como son los sistemas agroforestales; para esto entregó incentivos materiales, económicos y capacitación a productores. Tomando en cuenta a (Kaimowitz, 1996:XI), muchos proyectos de $\mathrm{MRN}^{1}$ se financian sin componentes de investigación, aduciendo que no son necesarios. Sin embargo, la experiencia demuestra que alguna investigación hace falta. A criterio de (Flores et al 2001:29) las investigaciones realizadas en SAF en Nicaragua han sido poco diseminadas.

1 MRN. Manejo de Recursos Naturales 
Tomando como base las experiencias promovidas e implementadas por el programa socioambiental y desarrollo forestal (POSAF) en unidades productivas de la subcuenca río Estelí, el presente trabajo tuvo como objetivo evaluar el éxito de tres sistemas agroforestales implementados, desde su clasificación, aportes socioeconómicos y ambientales a las familias beneficiadas. Esto permitirá que organizaciones y entidades del sector agropecuario y forestal cuenten con elementos útiles para la planificación y toma de decisiones acertadas en nuevas inversiones de proyectos similares.

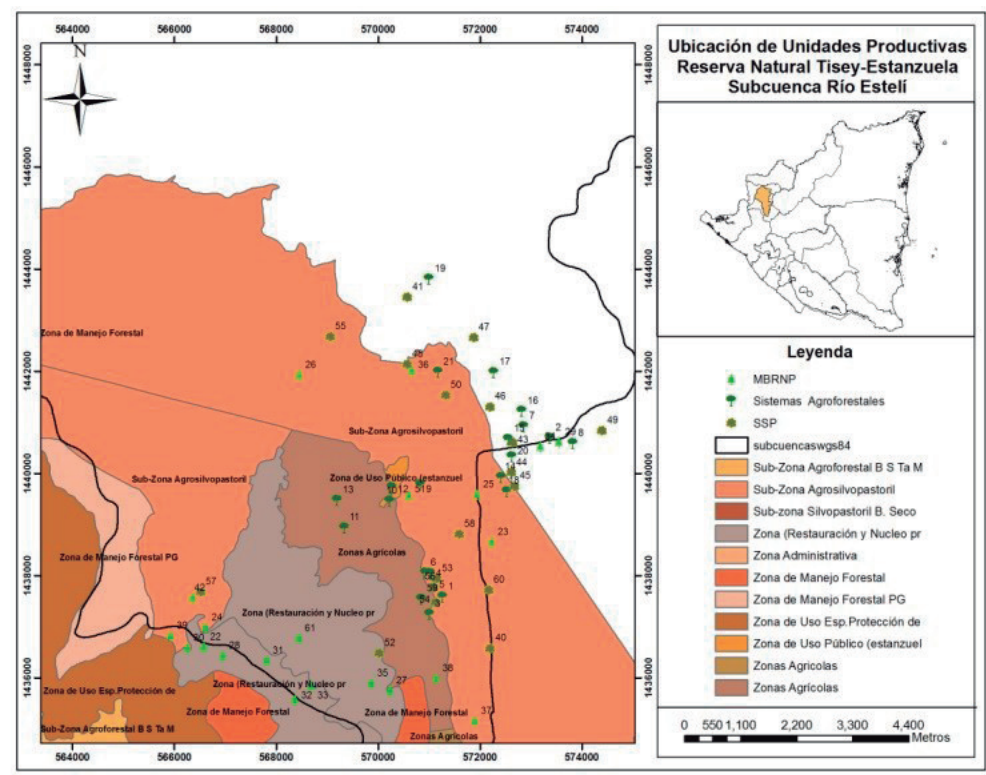

$86^{\circ} 17^{\prime} 34^{\prime \prime}$ longitud oeste (MARENA-POSAF, 2001). (INETER, 2013), reporta que para el periodo 2003 2013 la temperatura media anual fue de $24.6^{\circ} \mathrm{C}$ y la precipitación media anual fue de $910 \mathrm{~mm}$. Los rangos de elevación oscilan de 600 a 1.269 msnm (MARENAPOSAF, 2001).

Según Holdridge (1987) se clasifica como zona de vida de bosque Sub Tropical, transición a húmeda, Sub Tropical húmeda y Montano transición a húmedo. La población estimada en la parte alta de subcuenca del río Estelí es de 3059 habitantes (INIDE 2008). Es una subcuenca de prioridad muy alta por el profundo nivel de intervención agropecuaria e inadecuado manejo de los recursos naturales (MARENAPOSAF, 2001).

\section{Sistemas y tecnologías agroforestales promovidas}

En la tabla 1, se describen los sistemas agroforestales y tecnologías de conservación de suelo y agua promovidas por El Programa Socio ambiental y Desarrollo Forestal (POSAF II), fase 2006 al 2007.

Tabla 1. Menú de tecnologías y prácticas promovidas en unidades productivas, Subcuenca Río Estelí.

Figura 1. Ubicación de unidades productivas y tipo de sistemas agroforestales estudiados. Reserva Tisey

Estanzuela. 2013.

\section{MATERIALES Y MÉTODOS}

El estudio se realizó en la Reserva Natural TiseyEstanzuela, ubicada al suroeste del departamento de Estelí, Nicaragua; con extensión de 9,339.9 ha (MARENA-DGPN, 2009). Fue declarada Área Natural Protegida de Interés Nacional mediante Decreto Ejecutivo No.42-91 (MARENA-SINIA, 2010). La reserva se localiza en la parte alta de la sub cuenca del río Estelí, con una extensión de 1,326.50 $\mathrm{km} 2$., entre las coordenadas $13^{\circ} 13^{\prime} 33^{\prime \prime}$ latitud norte y

\begin{tabular}{|l|l|l|}
\hline $\begin{array}{c}\text { Tecnologías } \\
\text { promovidas } \\
\text { en sistemas } \\
\text { agrosilvícolas } \\
\text { (SAF) }\end{array}$ & $\begin{array}{c}\text { Tecnologías } \\
\text { promovidas } \\
\text { en sistema } \\
\text { silvopastoril (SSP) }\end{array}$ & $\begin{array}{c}\text { Tecnologías } \\
\text { promovidas en } \\
\text { manejo de bosque } \\
\text { con regeneración } \\
\text { más pasto } \\
\text { (MBRNP) }\end{array}$ \\
\hline Cercas vivas (m) & Cercas vivas (m) & $\begin{array}{l}\text { Cercas vivas y } \\
\text { Rondas corta fuegos } \\
\text { (m) }\end{array}$ \\
\hline $\begin{array}{l}\text { Plantaciones } \\
\text { Agroforestales } \\
\text { (ha) }\end{array}$ & $\begin{array}{l}\text { Plantaciones } \\
\text { silvopastoriles (ha) }\end{array}$ & $\begin{array}{l}\text { Reforestación } \\
\text { y plantación } \\
\text { industrial (ha) }\end{array}$ \\
\hline $\begin{array}{l}\text { Barreras vivas de } \\
\text { doble propósito } \\
\text { (m) }\end{array}$ & $\begin{array}{l}\text { Banco forrajero } \\
\text { de gramíneas y de } \\
\text { plantas forrajeras } \\
\text { (ha) }\end{array}$ & $\begin{array}{l}\text { Bancos forrajeros de } \\
\text { gramíneas (ha) }\end{array}$ \\
\hline $\begin{array}{l}\text { Establecimiento } \\
\text { de frutales } \\
\text { Injertos (ha) }\end{array}$ & $\begin{array}{l}\text { Establecimiento de } \\
\text { pasturas mejoradas } \\
\text { (ha) }\end{array}$ & $\begin{array}{l}\text { Bancos forrajeros de } \\
\text { plantas (ha) }\end{array}$ \\
\hline
\end{tabular}




\begin{tabular}{|l|l|l|}
\hline $\begin{array}{l}\text { Barreras vivas } \\
\text { arbustivas (m) }\end{array}$ & $\begin{array}{l}\text { Establecimiento de } \\
\text { frutales (ha) }\end{array}$ & $\begin{array}{l}\text { Establecimiento de } \\
\text { frutales (ha) }\end{array}$ \\
\hline $\begin{array}{l}\text { Cultivos de } \\
\text { cobertura (ha) }\end{array}$ & $\begin{array}{l}\text { Obras de } \\
\text { conservación de } \\
\text { suelo y agua (m) }\end{array}$ & $\begin{array}{l}\text { Manejo de la } \\
\text { regeneración } \\
\text { Natural con pasto } \\
\text { (ha) }\end{array}$ \\
\hline $\begin{array}{l}\text { Obras de } \\
\text { Conservación } \\
\text { de Suelo y Agua } \\
\text { (herramientas). } \\
\text { (m) }\end{array}$ & $\begin{array}{l}\text { Plan de manejo de } \\
\text { bosques latifoliados } \\
\text { y de pino; Recursos } \\
\text { Naturales (ha) y } \\
\text { (POA) }\end{array}$ \\
\hline $\begin{array}{l}\text { Otros incentivos entregados fueron: pies de cría de lombrices, } \\
\text { prácticas optativas, hornillas mejoradas, arados mecanizados, } \\
\text { incentivos económicos, reservorios de agua; capacitación y } \\
\text { asistencia técnica. }\end{array}$ \\
\hline
\end{tabular}

Fuente: Adaptado de informe final y reglamento operativo,

FIDER $^{2}$ - POSAF II, 2007.

\section{Muestreo}

A la población de 72 productores beneficiados, se aplicó muestreo probabilístico estratificado, determinando: 22 en sistemas agrosilvícolas (SAF), 18 en sistema silvopastoril (SSP) y 21 en sistema manejo de bosque con regeneración natural más pasto (MBRNP), sumando un total de 61 productores. Los criterios de selección fijados fueron: haber sido beneficiado por POSAF II, vivir en la finca en los últimos 6 años y voluntad de participar en esta investigación.

\section{Técnicas e instrumentos utilizados en el estudio}

Para este estudio se diseñó y aplicó una encuesta con preguntas abiertas y cerradas a productores beneficiados con sistemas agroforestales, permitiendo caracterizar y clasificar los sistemas dentro de las unidades productivas (UP). Así mismo, se realizaron recorridos por parcelas para valorar estado actual de sistemas, identificar los factores de éxito y fracaso que han limitado o favorecido su adopción y evaluar aspectos socioeconómicos y ambientales. En el recorrido, con GPS (etrex Legend H), se ubicó geográficamente cada una de las unidades productivas.

2 FIDER. Fundación de investigación y desarrollo rural (Organismo Coejecutor del POSAF )

\section{Análisis de la información}

Previo a la realización de pruebas estadísticas, así como para comprobar la normalidad de los datos y determinar qué tipo de análisis realizar, se aplicó la prueba de Kolmogorov Smirnov (K-S) a cada una de las variables que se les aplicó ANOVA.

En la clasificación de los sistemas se aplicó análisis discriminante multivariado con funciones canónicas (Hernández, R. et al., 1995) y para la variable producción de leche/día se realizó análisis de conglomerados. Los software utilizados para el procesamiento estadístico fueron, Infostat versión 2008, SPSS 18, complementado el ordenamiento de datos y análisis con Excel 2007.

\section{Variables evaluadas}

Para la clasificación de los sistemas agroforestales encontrados y tomando en cuenta su estructura y función, se seleccionó 24 variables relacionadas a las principales tecnologías agroforestales y prácticas complementarias de conservación de suelo implementadas por el programa. Para tener un mayor nivel de confianza en los resultados se definieron cuatro grupos de variables: Las tecnologías agroforestales promovidas y establecidas como: reforestación, cercas vivas, pasto mejorado, regeneración natural, plantación industrial, frutales establecidos y barreras vivas de gramíneas con doble propósito. Tecnologías que se continúan realizando después del proyecto siendo éstas: reforestación, cercas vivas, pasto mejorado, barreras vivas y regeneración natural. También se tomaron aquellas tecnologías y prácticas consideradas con mayor nivel de éxito, como: barreras vivas, cercas vivas, bancos forrajeros, manejo de bosques, frutales y regeneración natural y finalmente se incluyó variables de tecnologías que hacen aportes como: madera, postes, estacas prendedizas, frutas y forraje.

Otras variables socioeconómicas evaluadas fueron: ingreso agropecuario en dólares ${ }^{3}$, producción de leche

3U\$: dólares americanos. Se convirtieron utilizando la tasa de cambio de córdobas a dólares del Banco Central de Nicaragua. 
(1/vaca/día), cantidad de cabezas de ganado por sistema agroforestal, cantidad de aves de patio y consumo de leña (t/año).

\section{RESULTADOS Y DISCUSIÓN}

\section{Clasificación de los sistemas Agroforestales}

Los resultados muestran que la clasificación de los productores agrupados por sistema se ha realizado adecuadamente en el $93.4 \%$ de los casos. Sin embargo, 22 sistemas clasificados originalmente como agrosilvícolas, estadísticamente solo pertenecen 20 (90.9\%), 2 de éstos deberían estar agrupados dentro del sistema silvopastoril; de 18 productores en SSP, pertenecen $17(94.4 \%)$ y 2 se clasifican para el sistema MBRNP, y de los 21 sistemas MBRNP originales, quedan $20(95.2 \%)$ dentro de esta categoría y uno debería estar dentro del sistema agrosilvícola.

La figura 2 muestra la cohesión de los productores alrededor de cada sistema en función de las tecnologías agroforestales implementadas. En el sistema silvopastoril se observa mejor la relación de los productores alrededor de este sistema. Los datos de clasificación también indican que los sistemas tienen tecnologías agroforestales no adecuadas para ese sistema y que los incentivos materiales entregados y ciertas tecnologías agroforestales promovidas por el programa se entregaron indistintamente del sistema en forma de paquetes tecnológicos.

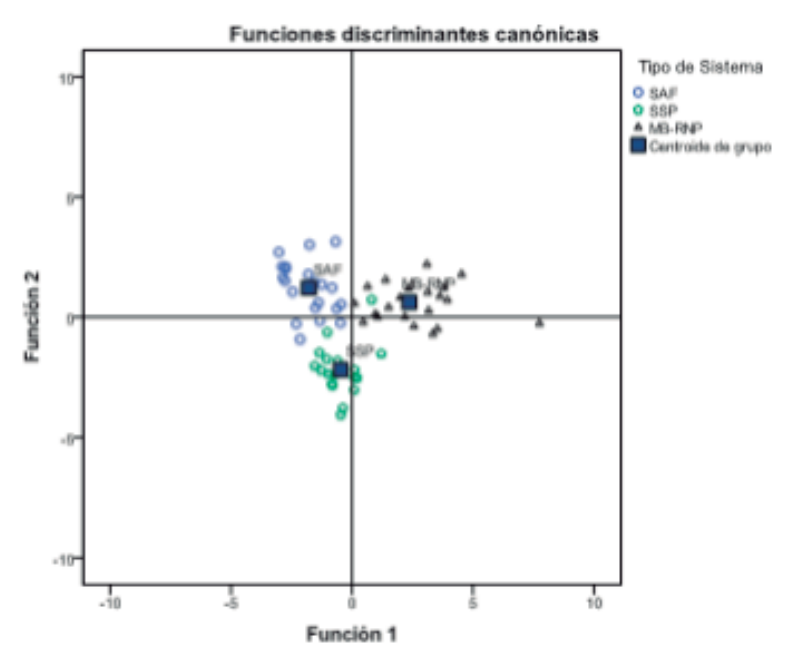

Figura 2. Clasificación de los productores de acuerdo a sistemas agroforestales. $n=61$
Adaptado de (Nair, 1997:27), desde su estructura y función los sistemas agrosilvícolas (SAF) encontrados en las unidades productivas, están compuestos principalmente por cultivos anuales, con arreglo de leñosas y cultivos de manera intermitente. Están dispuestos de manera lineal en las cercas vivas y en algunos casos árboles en linderos. Las funciones, primordialmente son la producción de alimentos, forraje, estacas prendedizas y leña. Las obras complementarias de conservación de suelo, tienen la función ambiental de disminuir la erosión de suelos e infiltración de agua. Las áreas de estas unidades productivas oscilan entre 1 a 3 y 4 a 7 ha en el 31.8 y $27.3 \%$ de productores respectivamente.

Económicamente (Lundgren, 1982 en Nair, 1997:33), clasifica los sistemas agroforestales en comerciales, intermedios y de subsistencia. De acuerdo a los pocos aportes identificados en los sistemas agrosilvícolas estudiados, se catalogan de subsistencia, porque el uso de la tierra está dirigido a satisfacer especialmente las necesidades básicas de la familia.

Los sistemas silvopastoriles (SSP) estudiados, en su estructura y función incluyen componentes de: ganado, pastos naturales, bancos forrajeros de gramíneas, árboles dispersos en potreros y cercas vivas. El tipo de arreglo es intermitente y las funciones productivas identificadas son: producción de forraje para el ganado, leña, postes y sombra. El 55.6\% tienen áreas entre 4 a 7 ha. De acuerdo a (Lundgren, 1982 en Nair, 1997:33), estos sistemas están entre la escala de subsistencia a intermedio, ya que aparte de satisfacer sus necesidades, tienen pequeños excedentes para la comercialización.

Los sistemas agrupados en manejo de bosques con regeneración natural más pasto (MBRNP), desde su estructura y función incluyen áreas de bosques, bosques y regeneración natural con pasto natural; bancos forrajeros de gramíneas; incluyen también áreas de cultivos y hortalizas. En caso de áreas con bosque y pastos, el tipo de arreglo encontrado es coincidente y en áreas de cultivos es intermitente. Las funciones 
productivas son: producción de madera, leña, postes, forraje para el ganado y alimento para la familia. Desde la escala económica se clasifican de subsistencia a intermedios. La cosecha es comercializada con intermediarios dentro de la comunidad o en mercados de Estelí. Los productores bajo este tipo de sistema (81\%) tienen áreas superiores a 12 ha y otros medios de vida.

Para los tres sistemas estudiados, el $80.3 \%$ tienen áreas de patio ${ }^{4}$, menores de una ha y ubicados cerca de la casa. Sus principales componentes son: ganado menor, plantas aromáticas de Menta piperita L. (hierbabuena) y Eryngium foetidum L. (chicoria) y en época lluviosa algunos cultivos de ramada; incluyen también árboles frutales y cercas vivas. Las funciones productivas son: alimento, sombra, protección y leña.

\section{Aportes socioeconómicos por tipo de sistema agroforestal}

\section{Ingresos Agropecuarios}

El sistema agrosilvícola (SAF) mostró diferencias estadísticamente significativas $(\mathrm{p}=0.0119)$, con ingresos menores en relación a los otros dos sistemas agroforestales, (Figura 3). Los ingresos agropecuarios promedios estimados por tipo de sistemas en el año 2012 son: sistema agrosilvícola (SAF) U\$ 103.59 \pm 30.83 dólares/mes, sistema silvopastoril (SSP) U\$ $170.31 \pm 29.13$ dólares/mes y con un mayor ingreso tenemos al sistema manejo de bosque (MBRNP) con U\$ $199.16 \pm 32.09$ dólares/mes. Estos ingresos superan el salario mínimo agropecuario aprobado legalmente (Ley 625) por el Ministerio del Trabajo en Nicaragua (MITRAB, 2012, 2013), de U\$ 71.50 y U\$ 99.16 dólares americanos/mes.

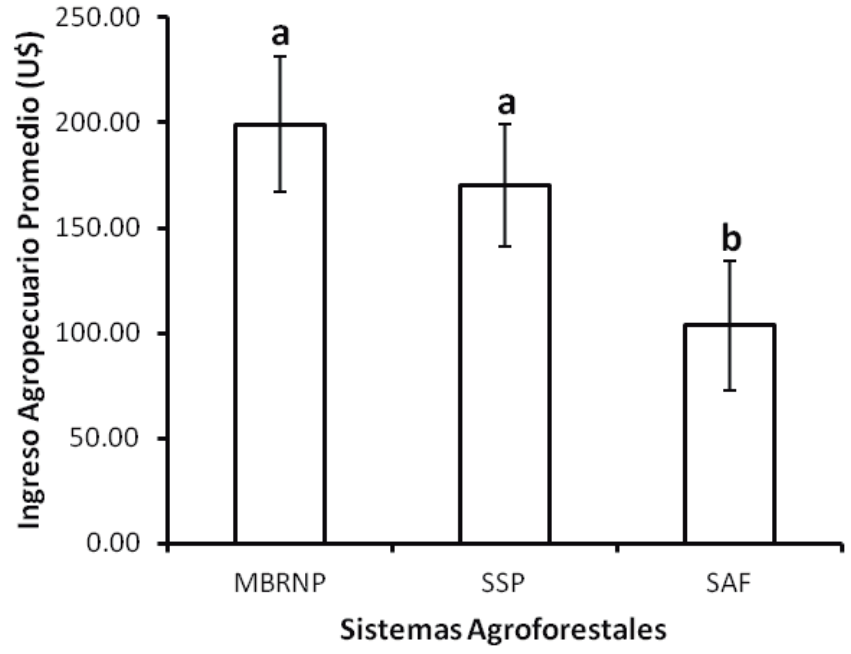

Figura 3. Ingreso agropecuario por tipo de sistema agroforestal $(1 \mathrm{U} \$$ Americano $=\mathrm{C} \$ 24.0773 . \mathrm{n}=45)$

(PRESANCA II, 2011), revela que la mayoría de países centroamericanos convergen hacia una canasta básica alimentaria entre 270 a 300 dólares americanos. Nicaragua y Honduras reportan un déficit de 100 dólares entre el salario mínimo y el costo de la canasta básica alimentaria, que al contrastarlo con ingresos agropecuarios de sistemas agroforestales estudiados el déficit es similar. Subrayar que $49 \%$ de las familias estudiadas dependen únicamente del ingreso agropecuario y son las familias agrupadas en sistemas agrosilvícolas las que dependen otros ingresos externos a la finca.

Para reducir este déficit las familias estudiadas tienen otros ingresos alternos como: remesas familiares (16 familias), recibiendo el $75 \%$ entre U\$ 50 a 100; 6 productores trabajan de jornaleros, recibiendo entre U\$42.56 a 136.20 dólares por mes; 10 familias tienen negocios propios, los ingresos para el 50\% de éstos fluctúa de U\$50 a 166 dólares y el 50\% restante obtienen entre U\$166.00 a 446.47 dólares/mes. Por último 24 productores declaran ingresos de otras fuentes, donde el 58.3\% oscila entre U\$16.62 a 103.83 dólares/mes.

4 Patio. Solares, huertos. 


\section{Producción de Leche}

El proyecto (FIDER-POSAF II, 2007), determinó que la ganadería en la zona es extensiva y se práctica en todo el área, principalmente ganado bovino con rendimientos muy bajos de leche (3 a 4 1/vaca/día), mayoritariamente utilizada para el autoconsumo. Actualmente los rendimientos promedios de leche, son inferiores a 2.91 1/vaca/día en época seca y 3.03 1/vaca/ día en época lluviosa. Los sistemas silvopastoriles y manejo de bosque con regeneración natural más pasto tienen un mayor rendimiento, con 5.81 1/vaca/día y 4.6 1/vaca/día respectivamente. Los rendimientos están influenciados por la cantidad de vacas de ordeño, nivel de manejo y áreas de pastoreo.

Tomando como referencia los datos de producción de leche diaria, período seco, con un precio de U\$ 0.49 dólares/litro, que es cuando baja la producción, muestra que no existen diferencias significativas en los ingresos diarios por producción de leche entre los sistemas $(\mathrm{p}>0.05)$. La Figura 4 muestra que hay una similitud entre sistema silvopastoril y manejo de bosque con regeneración natural más pasto, diferentes en ingreso por producción de leche/día del sistema silvoagrícola, éste ultimo sistema con reducidas áreas de pasto, menos cabezas de ganado y bajo manejo. Las características que los hacen similares son: cantidad de vacas de ordeño, tipo de manejo del hato e ingreso agropecuario. El ingreso promedio diario por producción de leche en sistema MBRNP es de U\$ $12.64 \pm 4.13$ dólares, en SSP es de U\$ $8.76 \pm 3.37$ dólares y para sistema agrosilvícola (SAF) es menor con U\$ $3.48 \pm 0.61$ dólares.

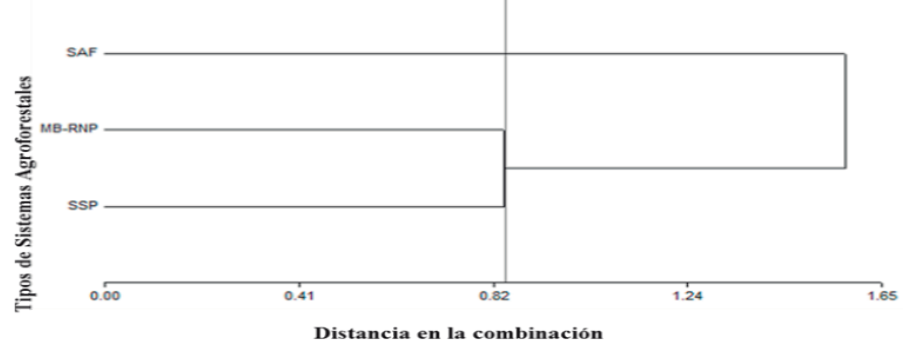

Figura 4. Dendrograma de ingreso de producción de leche/día. Estación de verano, $\mathrm{n}=33$

\section{Ganado mayor y menor}

El ganado mayor y menor para las familias, son parte de sus medios de vida, contribuyen a la seguridad alimentaria y nutricional. Al evaluar la cantidad de ganado por sistema se determina que existen diferencias significativas $(p=0.012)$, siendo los productores con sistema MBRNP (Figura 5) los que tienen mayor cantidad ( $28 \pm 7.20$ cabezas de ganado) y está relacionado a que 17 productores bajo este tipo de sistema tienen áreas superiores a 12 ha, disponiendo de mayores áreas de pastos. Seguidamente se ubica el sistema silvopastoril con $17.36 \pm 6.54$ cabezas de ganado y en último lugar los productores en sistema agrosilvícola con $10.93 \pm 3.60$ cabezas de ganado. La diferencia está estrechamente relacionada a la cantidad de áreas, manejo y recursos económicos de las familias.

De acuerdo a cantidad de aves de patio por sistema agroforestal, muestran que no existen diferencia estadísticamente significativas $(\mathrm{p}>0.05)$, siendo similares por tipo de sistema (Figura 6). Los sistemas agrosilvícolas (SAF) tienen en promedio 26.09 \pm 6.10 aves, sistema silvopastoril (SSP) con $21.73 \pm 4.36$ y las familias con menos aves son los agrupados en manejo de bosques (MBRNP), con $18.18 \pm 3.86$ aves. Este rubro de producción dentro de las unidades productivas esta a nivel de patios, con manejo extensivo y el uso es mayoritariamente para el autoconsumo, lo cual viene a complementar la dieta alimentaria.

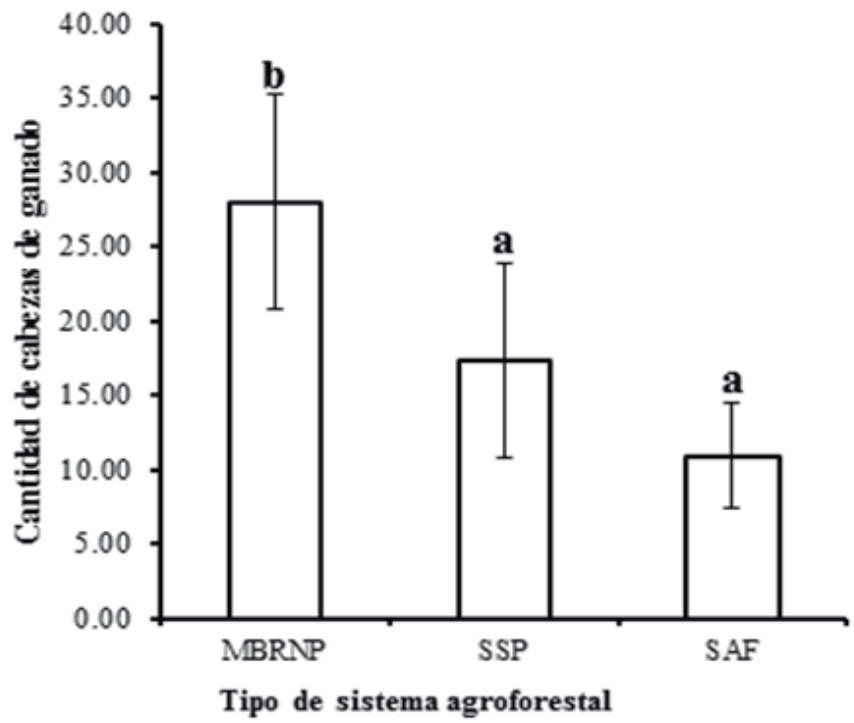

Figura 5. Cantidad de cabezas de ganado por tipo de sistema agroforestal. $n=42$ 


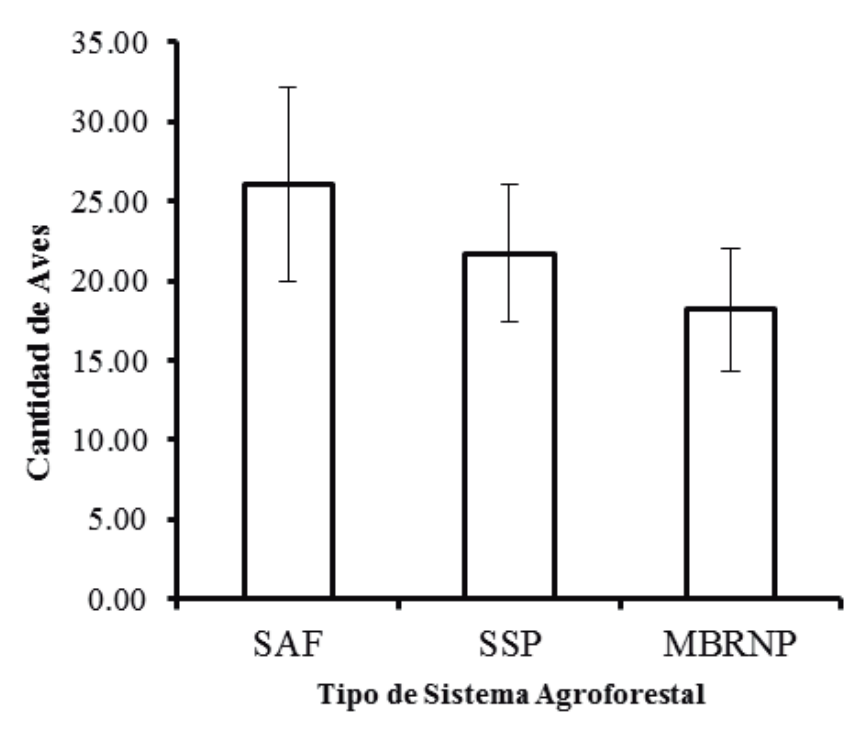

Figura 6. Cantidad de aves de patio por tipo de sistema agroforestal. $n=33$

El recurso forestal es complementario en un sistema agroforestal $\mathrm{y}$ hace aportes socioeconómicos $\mathrm{y}$ ambientales importantes a las familias, la leña es uno de los bienes aportados. Al analizar la cantidad de leña consumida anualmente por las familias agrupadas en los tres tipos de sistemas, estadísticamente no mostró diferencias significativas $(\mathrm{p}>0.05)$. El mayor consumo lo hacen familias con sistemas de manejo de bosque con $5.28 \pm 0.76 \mathrm{Tm} / \mathrm{año}$, seguido de familias en sistema agrosilvícola con $5.07 \pm 0.99 \mathrm{Tm} /$ anual y el menor consumo lo registran productores en sistema silvopastoril con $3.96 \pm 0.53 \mathrm{Tm} /$ año. Es decir que independientemente del tipo de sistema el consumo muestra un comportamiento similar. Un factor que podría estar incidiendo es que el núcleo familiar en promedio es de cuatro (4) personas. Resaltar que a pesar de que el programa implementó hornillas ahorradoras de leña en el año 2006, a la fecha, muy pocas familias las tienen, lo cual indica que la adopción de la tecnología fue baja y el nivel de consumo de leña actual es similar al que hacían las familias al inicio del proyecto (5.59 Tm/año).

\section{Tecnologías con mayor éxito en la implementación de sistemas agroforestales}

Las principales tecnologías agroforestales establecidas y con mayor nivel de éxito (Tabla 2) son: cercas vivas, plantación de frutales injertos, regeneración natural, y departe de productores con sistemas de manejo de bosque (MBRNP), hubo mayor éxito en manejo y conservación de bosques y plantación forestal. Este nivel de éxito está relacionado a tres atributos que mencionan (Krishnamurthy y Ávila., 1999): la productividad, sostenibilidad y adoptabilidad. (Ospina A., 2004), explica que las cercas vivas tienen gran importancia por las múltiples funciones desempeñadas y presentan amplia distribución en tierras tropicales. Es decir, es una tecnología agroforestal plenamente aceptada, conocida y practicada. Estos atributos mencionados por Ospina, han permitido una mayor aceptación y adopción de las cercas vivas en el programa, donde las principales funciones son: división de áreas, en menor escala la producción de estacas prendedizas y forraje. El manejo realizado se limita a podas y replantación de estacas prendedizas.

La plantación de frutales injertos fue masiva por el programa, pero según productores hubo muchas pérdidas $(60 \%)$. Actualmente, 26 productores en los tres tipos de sistemas manifiestan que fueron exitosas, están plantadas a nivel patio con el fin de darles manejo, que consiste en riego, limpieza y en algunos casos control de plagas y enfermedades. La principal función de éstas es la producción de frutas para el autoconsumo y únicamente tres productores reportaron venta en el mercado de Estelí. Las principales especies son Citrus ssp y Manguifera indica (L).

La regeneración natural se está realizando, principalmente en sistemas de manejo de bosques (MBRNP), que tienen mayor cantidad de áreas; a medida que van limpiando potreros seleccionan las especies arbóreas de interés. Las funciones de estas áreas son: sombra, forraje, leña y postes. El manejo se limita a chapias y selección de especies. 
Las áreas de manejo y conservación de bosques se limitan a productores con sistemas de MBRNP. Inicialmente con el programa se elaboraron 8 planes de manejo de bosques en 174 has y 6 planes operativos; en su momento se hicieron podas y cortas de saneamiento de madera de Pinus oocarpa Schiede ex Schltdl y Quercus segoviensis Liebm, actualmente los planes se vencieron, se hace aprovechamiento para uso interno de productores, ya que el $38 \%$ se ubican dentro del área núcleo de la reserva natural Tisey Estanzuela y hay restricciones para el corte de madera.

Los bancos forrajeros de gramíneas han tenido mayor éxito en sistemas silvopastoriles y sistema de manejo de bosque, algunos productores han multiplicado las áreas y la principal función es alimentación de ganado en época seca, que es cuando escasea el pasto. Las especies establecidas fueron: King grass, y Caña japonesa. Destacar que de las áreas de banco forrajero con leguminosas promovidas por el programa no se encontraron. A criterio de (Murgueitio E. et ál. 2006:367), la adopción, apropiación y multiplicación de los sistemas agroforestales por parte de los campesinos es un proceso dinámico, donde interactúan varios factores y que requiere tiempo, dedicación y esfuerzos de todo tipo.
Esto se evidencia en la adopción de obras de conservación de suelo y agua, como: barreas vivas, diques y barreas muertas, complementarias a los sistemas agroforestales promovidos. Las barreras vivas de gramíneas han tenido mayor aceptación y adopción por parte de 41 productores, $48.8 \%$ están dentro del sistema agrosilvícolas (SAF), debido a que el objetivo principal de éstas unidades productivas es la producción de granos básicos para la subsistencia. El $45.72 \%$ de los productores perciben que las barreras vivas están contribuyendo a la conservación de suelo y agua; además, en verano el $43 \%$ lo utilizan para la alimentación de ganado. Para (Pattanayak et al. 2002), la calidad pobre de los suelos y la amenaza de degradación se correlacionan positivamente con la adopción de prácticas de conservación de suelo y agua. En cada sistema y para las diversas tecnologías y prácticas agroforestales establecidas el manejo se limita a limpias, podas y en caso de productores que tienen áreas de café, únicamente se hacen chapias y regulación de sombra. A criterio de (Krishnamurthy \& Ávila, 1999), el manejo tiene como objetivo recuperar, aumentar o mantener el nivel de productividad de los sistemas agroforestales a mediano y largo plazo. Las técnicas empleadas para el manejo están orientadas a proteger el suelo de la erosión, mantener el ciclo de nutrientes, asegurar el suministro de agua y otros factores.

Tabla 2. Tecnologías agroforestales con mayor éxito por tipo de sistemas/subcuenca río Estelí, 2013.

\begin{tabular}{|c|c|c|c|}
\hline Tecnologías con mayor éxito & $\begin{array}{l}\text { Sistema agrosilvícola } \\
\text { (SAF) }\end{array}$ & $\begin{array}{l}\text { Sistema silvopastoril } \\
\text { (SSP) }\end{array}$ & $\begin{array}{c}\text { Manejo de Bosque con regeneración } \\
\text { natural más pasto (MBRNP) }\end{array}$ \\
\hline Cercas Vivas & $\begin{array}{l}18 \text { productores } \\
55 \% \text { con } 250 \text { a } 500 \\
\mathrm{~m} .\end{array}$ & $\begin{array}{l}13 \text { productores } \\
50 \% \text { tienen de } 100 \text { a } 500 \\
\mathrm{~m} .\end{array}$ & $\begin{array}{l}14 \text { productores } \\
50 \%(250 \text { a } 500) \\
28 \%(\text { menos de } 100 \mathrm{~m}) \\
\end{array}$ \\
\hline Frutales & $\begin{array}{l}10 \text { productores } \\
(0.01 \text { a } 0.25 \text { ha. })\end{array}$ & $\begin{array}{l}7 \text { productores } \\
(0.01 \text { a } 0.5 \text { ha. })\end{array}$ & $\begin{array}{l}9 \text { productores } \\
75 \% \text { (áreas de } 0.01 \text { a } 0.50 \text { ha) }\end{array}$ \\
\hline Regeneración Natural & $\begin{array}{l}5 \text { productores Áreas } \\
\text { menores a } 0.5 \text { ha }\end{array}$ & $\begin{array}{l}7 \text { productores } \\
62.5 \% \text { menores de } 0.50 \text { ha }\end{array}$ & $\begin{array}{l}11 \text { productores } \\
42 \% \text { ( } 1 \text { a } 4 \text { ha })\end{array}$ \\
\hline Barreras Vivas de Gramíneas & $\begin{array}{l}20 \text { productores } 55 \% \\
(251 \text { a } 500 \mathrm{~m} .)\end{array}$ & $\begin{array}{l}11 \text { productores } \\
45.55 \% \text { (251 a } 500 \mathrm{~m} .) \\
27.3 \% \text { (más de } 500 \mathrm{~m})\end{array}$ & $\begin{array}{l}10 \text { productores } \\
54.5 \%(0 \text { a } 250 \mathrm{~m}) \\
45 \%(250 \text { a } 500 \mathrm{~m})\end{array}$ \\
\hline $\begin{array}{l}\text { Bancos Forrajeros de } \\
\text { Gramíneas }\end{array}$ & & $\begin{array}{l}12 \text { productores } \\
44 \% \text { áreas entre } 0.5 \text { a } 1 \text { ha }\end{array}$ & $\begin{array}{l}12 \text { Productores } \\
50 \%(0.17 \text { a } 0.70 \text { ha }) \\
37.5 \%(1 \text { a } 3.5 \mathrm{ha})\end{array}$ \\
\hline Diques & 11 productores & 15 productores & 10 productores \\
\hline Barreas Muertas (productores) & 10 productores & 9 productores & 6 productores \\
\hline
\end{tabular}


Para los tres tipos de sistemas agroforestales promovidos, las cuatro principales limitantes asociadas a pérdidas de los materiales vegetativos (frutales, plantas forestales, cercas vivas) reportados por productores y observados en campo fueron: afectación por sequía o exceso de agua (21.31\%), materiales vegetativos no aptos para la zona, de baja calidad y entregados tardíamente $(21.31 \%)$, poco interés, negligencia de los productores y desconocimiento de manejo (19.67\%) y daños mecánicos causados por ganado $(18.3 \%)$.

A nivel de tecnologías agroforestales y a criterio de los productores, las plantaciones agroforestales, silvopastoriles y enriquecimiento de bosques; plantas frutales y estacas prendedizos en cercas vivas son las que mostraron mayores pérdidas. (Clavero y Suárez, 2006:1; Pattanayak et al. 2002), mencionan que algunas de las causas principales que han limitado el desarrollo de las tecnologías agroforestales son: factores técnicos como germoplasma, problemas de plagas y enfermedades, información técnica relativa a producción y calidad, incentivos de mercado, biofísicos, investigaciones no orientadas y falta de educación agroforestal. Por su parte (Toruño P. J. et al., 2012:5), describe que un factor a considerar de los sistemas agroforestales, es que el cambio en el sistema productivo ocurre a largo plazo, los beneficios obtenidos por éstos no resuelven la situación actual a la que se enfrentan los productores.

Visto desde los sistemas agroforestales promovidos, el fracaso y abandono de algunas tecnologías y prácticas agroforestales está relacionado a que la producción y beneficios de los sistemas son a mediano y largo plazo, lo cual desestimula a beneficiarios que quieren resolver las necesidades económicas y alimenticias de cada día. Esta actitud de abandono, podría estar vinculada a un nivel bajo de capacitaciones y entrenamiento para establecer y darle un manejo eficiente a cada una de las practicas. (Snook y Zapata, 1998) en evaluación de proyecto agroforestal encontró un alto nivel de abandono de las parcelas post proyecto por parte de los productores; identificando las siguientes limitaciones: falta de apoyo técnico, calidad de las plantas y falta de productos inmediatos.

El razonamiento de (Ospina A., s.f), es que la adopción de sistemas agroforestales novedosos no es fácil en comunidades rurales. Debe trabajarse preferiblemente en la mejora, sofisticación y fortalecimiento de las ya existentes y útiles, donde se involucren actividades compatibles con la economía y cultura. Algunas claves propuestas por (Murgueitio, 2006:5), para implementar sistemas agroforestales pecuarios son: multiplicación del material vegetal apropiado y adaptado a cada zona, identificar opciones de comercialización, acompañamiento técnico y capacitación para el cambio cultural, experimentación local y sistematización permanente.

A nivel de unidades productivas estudiadas y en entrevista con productores se evidencia que de acuerdo a sistemas agroforestales promovidos, hay mayor adopción y sostenibilidad de algunas tecnologías agroforestales y prácticas de conservación de suelo. (Clavero y Suárez, 2006:1), expresan que para lograruna adopción exitosa y entusiasta sistemas agroforestales se requiere de una real integración entre productores, investigadores y extensionistas, alta capacidad de ejecutar innovaciones tecnológicas y organizativas para difundir y adoptar, no sólo proyectos adaptados a una determinada zona agroecológica, sino también a las condiciones y características del productor, el cual tiene que desempeñar un papel activo para lograr elevadas tasas de adopción y resultados exitosos. Este éxito de la agroforestería, a criterio de (Casanova, et al. 2011:141), implica o se traduce en beneficios sociales, ecológicos y económicos. 


\section{Principales aportes ambientales de los sistemas agroforestales.}

Los principales aportes ambientales identificados por productores, 6 años después de finalizado el programa y relacionados a sistemas agroforestales establecidos son: $100 \%$ de productores con tecnologías y prácticas de conservación de suelo y agua reducen la erosión de suelos y aumentan la infiltración del agua; así mismo productores manifiestan que producto de la conservación de suelo han reducido al $50 \%$ el uso de fertilizantes. (White y Jickling, 1994, en Kaimowitz, 1996:X), indican que hay prácticas sencillas que conservan el suelo, demandan pocos recursos y aportan múltiples beneficios concretos a corto plazo; que de acuerdo a (Duarte, 2005 en Arias y Camargo, 2007:16), cuanto mayor sea el uso de estas prácticas mayor es la tendencia hacia la estabilidad y sostenibilidad ecológica de los sistemas de producción.

El $61.8 \%$ de los productores identifica que hay más sombra para el ganado y para las familias, dotando de un microclima agradable en la unidad productiva; 93.4\% de los productores expresaron no hacer quemas agrícolas, lo que se traduce en menos aportes de CO2 a la atmosfera. (Beer, et ál. 2004 en Saravia, 2009:231) exponen que la presencia de árboles en sistemas agrícolas tradicionales tienen muchos propósitos como producción (madera, leña, forraje, frutas, medicinas, etc.), brindan servicios (sombra para cultivos $\mathrm{y} / \mathrm{o}$ animales, protección como en el caso de cortinas rompevientos, etc), además, los árboles aumentan la diversidad biológica creando en sus ramas, en sus raíces y en la hojarasca, hogares para otros organismos.

En relación a lo planteado, (Murgueitio, 2009:12), describe que otros resultados positivos del proceso de adopción de sistemas silvopastoriles y protección de bosques en un proyecto ejecutado fueron: incremento en la biodiversidad y captura de carbono en el corto plazo en tierras agropecuarias. Por su parte (Pomareda, 2001; Giraldo, 1996 y Pérez, 2006) sostienen que los sistemas silvopastoriles con especies arbóreas dispersas, permiten dar un buen manejo al suelo y alberga una gran diversidad de flora y fauna. (Ibrahim, Camero, Camargo, \& Andrade, 2008), confirman que en estudios realizados en Costa Rica, se determinó que las especies forestales encontradas en potreros se usan principalmente como sombra para los animales o para madera, postes, leña, rompevientos o alimentos para aves.

\section{CONCLUSIONES}

Los productores agrupados en sistema agroforestal manejo de bosque con regeneración natural más pasto, presentaron los mayores aportes socioeconómicos en ingreso agropecuario y mayor cantidad de cabezas de ganado, sin embargo, hay mayor eficiencia en la producción de leche/ vaca/día en sistemas silvopastoriles y éstos, económicamente dependen menos de ingresos externos a la finca.

De acuerdo a ingresos económicos y el nivel de producción reportados por familias con sistemas agrosilvícolas se clasifican de subsistencia. Por estas razones dependen más de ingresos económicos externos a la finca como remesas, jornales y negocios propios.

Los productores agrupados en sistema silvopastoril presentaron los mayores niveles de éxito y adopción de tecnologías agroforestales en: Cercas vivas (m), áreas con frutales (ha), cantidad de diques, áreas de pasto y banco forrajero.

Extender este tipo de investigación en sistemas agroforestales post proyecto, a otras subcuencas atendidas por iniciativas de desarrollo rural, comparar los resultados y así robustecer nuevas decisiones e inversiones que se hagan. 


\section{AGRADECIMIENTOS}

A la Fundación Autónoma Solidaria de la Universidad Autónoma de Barcelona (UAB) y al Programa MARENA-PIMCHAS, por su colaboración técnica y financiera, posible gracias a la cooperación de los Pueblos y Gobiernos de Canadá y Nicaragua mediante un memorando de entendimiento firmado entre ambos países.

Al doctor Jordi Bartolomé de la UAB, por la revisión del manuscrito.

Al maestro Willian Ortiz por su valioso y oportuno aporte en los análisis estadísticos.

A los productores y productoras que me atendieron en cada una de las unidades productivas.

\section{BIBLIOGRAFÍA}

Araica C. R. \& Ruíz G.G. (2005). Determinación de Insectos y Patógenos en la Reserva Natural Meseta Tisey- Estanzuela, Estelí. Trabajo de diploma. Managua. UNA. 55 pág.

Arias-Giraldo L. M.; Camargo J. C. (2007). "Sustainability analysis for cattle-farms in the municipality of Circasia (Quindío state, Colombia), La Vieja river watershed". En línea. Revista Livestock Research for Rural Development 19 (10). Disponible en http://www.lrrd.org. Consultado el 16 de julio 2013.

Banco Central de Nicaragua BCN (2013). Tipos de cambio oficial del córdoba con respecto al dólar de los estados unidos de América 2012. Consulta en línea. Disponible en http://www.bcn.gov.ni. Consultado el 10/06/2013. Managua-Nicaragua.

Casanova L. F. (2011). Agroforestry systems as an alternative for carbon sequestration in the Mexican tropics. Revista Chapingo Serie Ciencias Forestales y del Ambiente 17(1): 5-118, 2011.Disponible en http:// www.chapingo.mx./revistas. Consultado el 15 de Junio del 2013.
CEPAL (2003). Istmo Centroamericano: Los Retos de la Sustentabilidad en Granos Básicos. ONUCEPAL, México. 96 pág.

Clavero, T. \& Suárez, J. (2006). Limitaciones en la adopción de los sistemas silvopastoriles en Latinoamérica. Pastos y Forrajes, Vol. 29, No. 3. p. 307.

Current, D. (1995). Adopción agrícola y beneficios económicos de la agroforestería: Experiencia en América Central y El Caribe. Serie Técnica. Informe Técnico 268. CATIE. Turrialba, C.R. 1995. 39 pág.

FAO. (s.f). Sistemas Agroforestales en América Latina y El Caribe. Oficina Regional. 94 pág.

FIDER, (2003). Plan de Manejo Reserva Natural Tisey-Estanzuela. Estelí Nicaragua. 288 pág.

FIDER-POSAF II, (2007). Informe final del proyecto "Manejo Sostenible de los Recursos Naturales y Fortalecimiento de las Capacidades Socioeconómicas de la Población de la Sub-cuenca Sur del Río Estelí y la Microcuenca El Jicarito del Municipio de Yalagüina". BID - MARENA / POSAF II. Estelí, Nicaragua. 39 pág.

Flora Mesoamericana. Consulta en línea. Disponible en http://www.tropicos.org/ Name/24900645 projectid=3\&langid=66. Fecha de Consulta 20 de Mayo del 2013.

Flores E., Soriano D., Harvey C., López J., (20 0 1). Caracterización de la investigación agroforestal en el Pacífico de Nicaragua durante el decenio 19891999. Revista Agroforestería en las Américas. Vol .8 No...3 , pág. 25 a 30. Catie, Costa Rica.

Hernández Sampieri, R., Fernández Collado, C., \& Baptista Lucio, P. (1995). Metodología de la Investigación. Naucalpan de Juárez, México: McGraw- Hill. Cuarta Edición.

Holdrige, L. 1987. Ecología basada en zonas de vida. Tercera edición. Instituto interamericano de cooperación para la agricultura (IICA),San José, Costa Rica. 216 p.

Ibrahim, M. et al. (2007). Sistemas Silvopastoriles como una herramienta para el mejoramiento de la productividad y rehabilitación ecológica de 
paisajes ganaderos en Centro América. Archivos Latinoamericanos de Producción Animal. Vol. 15, 74-88.

Instituto Nicaraguense de Estudios Territoriales, INETER (2013). Resumen meteorológico anual. Estación meteorológica de Condega/Estelí. Código 45050, tipo AG. Nicaragua.

Instituto de Información y Desarrollo, INIDE (2008).

Estelí en Cifras. URL: www.inide.gob.ni. (Consultado en línea, 10 de Junio del 2013). Managua, Nicaragua. 79 pág.

Instituto de Información y Desarrollo INIDE (2013).

Costo de la Canasta Básica Alimentaria en Nicaragua. Disponible en http://www.inide.gob.ni/ CanastaB/CanastaB.htm (consultado 15 de Abril 2013). Managua, Nicaragua. 2 pág.

Instituto de Información y Desarrollo INIDE (2012).

Costo de la Canasta Básica Alimentaria en Nicaragua. Disponible en http://www.inide.gob.ni/ CanastaB/CanastaB.htm (consultado 15 de Abril 2013). Managua, Nicaragua. 2 pág.

Krishnamurthy L., Ávila M. (1999). Agroforestería Básica. Red de Formación Ambiental. Serie Textos Básicos para la Formación Ambiental. ISBN 9687913-04-5. D.F. México. 340 pág.

MARENA- SINIA, (2008). GEO, IV informe del estado del Ambiente. Nicaragua. Período 20072008. 339 pág.

MARENA-SINIA, (2010). El Sistema de Monitoreo y Evaluación Integral de Efectividad de Manejo en las áreas protegidas del SINAP. Disponible en URL: http://www.sinia.net.ni/wsinap/component/ content/article/59. (Consulta en Línea, 21 de Junio del 2013).

Ministerio del Trabajo MITRAB (2007). Ley 625 o Ley del salario mínimo. Gaceta Diario Oficial número 120. Managua Nicaragua.

Ministerio del Trabajo MITRAB (2013). Acta número uno sobre salario mínimo. Comisión Nacional de
Salario Mínimo (CNSM). Disponible en http:// www.mitra.gob.ni/documentos/salariominimo, consultado el 15 de Junio del 2013.

Montagnini, F. (1992). Sistemas agroforestales: principios y aplicaciones en los trópicos. 2 ED. San José. Organización para estudios tropicales. 622 pág.

Murgueitio E. (2009). Incentives for silvopastoral systems in Latin America. Avances de Investigación. 13(1): 3-19. CIPAV , Colombia.

Murgueitio E., et ál. (2006). Adoption of Agroforestry Systems for Animal Production. Revista Pastos y Forrajes. Vol. 29, No. 4, CIPAV.

Nair P.K.R. (1997) Agroforestería. Centro de Agroforestería para el desarrollo sostenible, Universidad Autónoma de Chapingo, Chapingo, Estado de México.543 p.

Ospina A. (1995). Características agroforestales de las huertas familiares. Cali, Colombia.

Ospina A. (2004).Cerca viva. Disponible en www. ecovivero.org. Consultado en línea el 20/06/2013.

Palacios P., Martínez N. (s.f). La agroforestería comunitaria en Santa María Huatulco Oaxaca, México. GAIA. 7 p.

Saravia P. M. (2009). Sistema silvoagrícola en la comunidad de Iscaypata, municipio de Vinto. Revista ACTA NOVA; 1er Congreso Nacional de Sistemas Agroforestales. Vol. 4, No 2-3, PROMIC, Cochabamba, Bolivia.

Snook, A. and G. Zapata (1998). 'Tree cultivation in Calakmul, Mexico: alternatives for reforestation, Agroforestry Today 10:15-18.

Toruño P. J. et ál. (2012). Acceptability and Adoption of Adaptative Production Systems in The Watershed "El Guayabo-San José", El Sauce Municipality, Leon. Revista Científica La Calera. Vol. 12. № 18, p. 52-60 / junio 2012. 